This article is licensed under the Creative Commons Attribution-NonCommercial 4.0 International License (CC BY-NC) (http://www.karger.com/Services/OpenAccessLicense) Usage and distribution for commercial purposes requires written permission.

\title{
Chronic Autoimmune Meningoencephalitis and Periodic Fever Syndrome Treated with Anakinra
}

\author{
Alyssa-Rae Novroski Kelly J. Baldwin \\ Department of Neurology, Geisinger Medical Center, Danville, PA, USA
}

\section{Keywords}

Meningitis - Encephalitis · Autoimmune meningoencephalitis · Anakinra · Periodic fever syndrome

\begin{abstract}
We report the case of a 63-year-old male diagnosed with chronic autoimmune mediated meningoencephalitis and periodic cyclical fever syndrome. After 2 years of failed conventional treatment and recurrent hospitalizations, anakinra treatment (an interleukin-1 [IL-1] receptor antagonist) was trialed which resulted in full recovery. As evidenced by this case, anakinra can be used to treat chronic autoimmune meningitis, which can often be refractory to treatment.

(C) 2017 The Author(s)

Published by S. Karger AG, Basel
\end{abstract}

\section{Introduction}

Chronic meningitis, defined by at least 1 month of unresolved inflammation of the cerebrospinal fluid (CSF), is a difficult illness to diagnose and treat [1]. Regardless of cause, pre- 


\section{Case Reports in Neurology}

Case Rep Neurol 2017;9:91-97

DOI: $10.1159 / 000472147$

(C) 2017 The Author(s). Published by S. Karger AG, Basel www.karger.com/crn

Novroski and Baldwin: Chronic Autoimmune Meningoencephalitis and Periodic Fever Syndrome Treated with Anakinra

senting symptoms can include headache, nausea/vomiting, cranial neuropathies, or other focal neurological deficits [1]. After a comprehensive history and physical examination, the initial step in narrowing the etiology is done via lumbar puncture (LP). CSF analysis should include laboratory studies for infectious, neoplastic, and autoimmune causes of meningitis. Autoimmune etiologies may present a particular challenge as patient presentations can vary widely and diagnostic workup is often nonspecific [1]. Patients frequently undergo repeat testing which is similarly unrevealing, causing frustration to both patient and practitioner. The pursuit of diagnosis may ultimately lead to more invasive testing such as brain biopsy. Treatment for autoimmune meningoencephalitis includes steroids and immunosuppressive agents, but unfortunately their success rate is variable [1]. Nonetheless, reducing morbidity and mortality relies on early diagnosis and treatment, and can require an interdisciplinary approach with regard to patient care, as exemplified in detail below [1].

\section{Case Presentation}

A 63-year-old male with a past medical history of remote facial trauma presented to our hospital with a 1-month long history of fever, headache, and gait instability. The headaches were left sided, constant, and throbbing with associated nausea and vomiting that was worse in the morning. During that month, he also developed progressive difficulty with balance and a fine tremor in his bilateral upper extremities which worsened with activity. He complained of daily chills and was found to be febrile on presentation. On physical exam, he was noted to have mild ataxia with finger-to-nose testing in both arms and inability to perform tandem walking. Neurologic exam was otherwise without focality or meningeal signs. Laboratory studies were only significant for nonspecific markers of inflammation such as C-reactive protein (CRP) $(92 \mathrm{mg} / \mathrm{L})$ and erythrocyte sedimentation rate $(37 \mathrm{~mm} / \mathrm{h})$. Magnetic resonance imaging (MRI) of the brain with and without gadolinium demonstrated scattered nonspecific T2 hyperintensities in bilateral cerebral white matter, without abnormal enhancement, as well as maxillary sinus disease. His headache was treated with steroids, ketorolac, and anti-emetics with mild improvement. His fever was not noted again in his hospital course.

Two months later, he presented again with continuing intermittent episodes of headache, fever, unsteady gait, and vomiting. He underwent a LP at this time which showed elevated protein, pleocytosis with neutrophilic predominance, and normal glucose. He was started on empiric antibiotics for meningitis. Extensive workup for bacterial, fungal, viral, and autoimmune etiologies was negative during this hospitalization, as well as subsequent hospitalizations. CSF studies included basic studies (Table 1) as well as testing for acid-fast bacilli stain, anaerobic culture, Aspergillus galactomannan, cryptococcal antigen, cytomegalovirus, enterovirus, fungal culture, group B Escherichia coli, Haemophilus influenza type B, herpes simplex virus 1 and 2, JC virus, Listeria antibody, multiple sclerosis IgG synthetic rate, Streptococcus pneumonia antigen, Tropheryma whippelii, varicella zoster, venereal disease research lab test (VDRL), and 14-3-3 protein. Hematologic infectious workup included blood cultures, complete blood count, antistreptolysin 0 titer, blastomycoses antibody, Brucella IgG, Coccidioides immitis, diphtheria, Epstein-Barr virus, Ehrlichia antibody, fluorescent treponemal antibody absorbed test (FTA-ABS), hepatitis C, human herpes virus 6 (HHV-6), 


\section{Case Reports in Neurology}

Histoplasma galactomannan antibody, HIV 1 and 2, Listeria antibody, Lyme antibody, mumps IgG antibody, Mycobacterium tuberculosis, pneumococcal antibody, procalcitonin, rapid plasma regain (RPR), and toxoplasma IgG, which were all negative. Hematologic autoimmune and neoplastic workup included anti-nuclear antibodies (ANA), anti-neutrophil cytoplasmic antibody (P-ANCA, C-ANCA), cyclic citrullinated peptide (CCP) antibody, complement C3 and C4, cryoglobulin screen, IgA, IgG, IgM, N-methyl-D-aspartate (NMDA) receptor antibody, rheumatoid factor (RF), serum protein electrophoresis (SPEP), SSA/SSB antibody, and voltage-gated potassium channel antibody, which were similarly unremarkable. This workup included paraneoplastic testing for antineuronal nuclear antibody types 1-3, antiglial nuclear antibody type 1, Purkinje cell cytoplasmic antibody type 1-2 and TR, amphiphysin antibody, CRMP-5 IgG, striational antibody, P-Q type calcium channel antibody, Ntype calcium channel antibody, and acetylcholinesterase receptor-binding antibody. MRI with and without gadolinium was repeated showing previously noted T2 hyperintensities as well as new areas of hyperintensities in the right cerebral hemisphere, right caudate, and the brainstem without abnormal enhancement. His symptoms improved with antibiotic therapy and he was discharged with a peripherally inserted central catheter (PICC line) to complete a treatment course of meropenem for presumed atypical bacterial meningitis.

Over the subsequent year, he returned to the hospital on a monthly basis with worsening fever, headache, tremor, gait instability, as well as an intermittent diffuse macular rash. As time progressed behavioral changes, including abulia and apathy, were becoming problematic. Repeat MRI with and without gadolinium at this point demonstrated diffuse T2 hyperintensities in both cerebral hemispheres, basal ganglia, and brainstem, as well as new bilateral leptomeningeal enhancement (Fig. 1). Repeat LPs consistently showed substantial pleocytosis and elevated protein (Table 1).

Other extensive workup was pursued including vascular imaging of his head and neck, CT chest, abdomen, and pelvis, temporal artery biopsy, and brain biopsy, which were nondiagnostic. Dilated ophthalmologic exam did not show any evidence of uveitis. Repeated treatments with broad-spectrum antibiotics did not improve his course and the patient's condition continued to deteriorate over the course of 1 year, leading to progressive decline in functional status. He was continually readmitted with a cyclical presentation of fever, each time at a worse baseline. On his final admission, he was significantly altered and found to be in generalized nonconvulsive status epilepticus. At this juncture, without a known infectious or neoplastic etiology confirmed, the team suspected that an autoimmune etiology may be causative. Steroid and antiepileptic treatment was initiated which caused nearly immediate improvement in the patient's mental status.

Unfortunately, despite improvement in mental status and behavior, he continued to have periodic flares of fever and rash nearly every 2 weeks. Over the next several months, intravenous immunoglobulin and mycophenolate were trialed; however, the patient continued to be readmitted on a monthly basis.

After failing these typical treatments for autoimmune meningoencephalitis, an interleukin-1 (IL-1) receptor antagonist called anakinra was trialed in this patient. The choice for this medication was mainly due to his cyclical fever/rash presentation, despite genetic testing being negative for periodic fever syndromes, including ELANE, LPIN2, MEFV, MVK, NLRP3, PSTPIP1, and TNFRSF1A. Remarkably, after being started on anakinra our patient experienced resolution of his meningoencephalitis, fevers, and rash. Over time, his other 


\section{Case Reports in Neurology}

Novroski and Baldwin: Chronic Autoimmune Meningoencephalitis and Periodic Fever Syndrome Treated with Anakinra

neurologic symptoms such as tremor, gait, abulia, and apathy also improved. His CRP and erythrocyte sedimentation rate, both of which remained elevated throughout his many hospitalizations, had normalized. His follow-up MRI of the brain with and without gadolinium following 1 year of treatment demonstrated resolution of the basal ganglia and midbrain hyperintensities and resolution of all leptomeningeal enhancement. Given the clinical and radiographical improvement, repeat LP was not done.

\section{Discussion}

Cyclical fevers are often a part of a class of diseases of innate immunity, called autoinflammatory diseases, in which the immune system activates without a driving antigen and generally lacks autoantibodies [2, 3]. Thus, diagnostic workup may initially only show elevation in nonspecific markers of inflammation (e.g., CRP, erythrocyte sedimentation rate) [4]. Autoinflammatory disorders should be suspected with recurrent unexplained manifestations of inflammation (arthritis, rash, meningitis, uveitis, serositis) [2, 3]. When cyclical fevers are present as well, these diseases are termed periodic fever syndromes $[3,4]$. They usually present in children who have recurrent fevers over months or years in the absence of infection, but can also present in adults [3].

Periodic fever syndromes are thought to be impelled in part by IL-1, a proinflammatory cytokine produced predominantly by macrophages and contributes to proteolysis and fever [5]. There are numerous periodic fever syndromes. Despite variation in their genetic mutations and affected proteins, many cause over activation or lack of suppression of the IL-1 pathway [2]. Anakinra acts as a competitive inhibitor of the interleukin- $1 \alpha$ and $-1 \beta$ receptors, thereby preventing signal transduction and mitigating the inflammatory response $[5$, 6]. It was originally used as a disease modifying antirheumatic drug in severe rheumatoid arthritis, but is more recently being used to treat periodic fever syndromes given the aboveproposed mechanism $[2,6]$. Specifically, cryopyrin-associated periodic syndromes, which increase IL-1 secretion due to defects of the NLRP3 gene, were among the first to show good clinical results when treated with IL-1 blockers [2].

After nearly 2 years from initial presentation, this patient has now achieved full remission. He is back to work as a farmer with no further hospitalizations. Based on this case, we propose that IL-1 inhibitors may be considered in a patient presenting with chronic autoimmune meningoencephalitis and periodic fever syndrome when other standard treatments have failed to relieve symptoms.

\section{Statement of Ethics}

The authors have no ethical conflicts to disclose.

\section{Disclosure Statement}

There are no conflicts of interest to disclose. 
Novroski and Baldwin: Chronic Autoimmune Meningoencephalitis and Periodic Fever Syndrome Treated with Anakinra

\section{References}

1 Baldwin KJ, Zunt JR: Evaluation and treatment of chronic meningitis. Neurohospitalist 2014;4:185-195.

-2 Goldbach-Mansky R: Immunology in clinic review series; focus on autoinflammatory diseases: update on monogenic autoinflammatory disease: the role of interleukin (IL)-1 and an emerging role for cytokines beyond IL-1. Clin Exp Immunol 2012;167:391-404.

-3 Federici S, Sormani PM, Ozen S, et al: Evidence-based provisional clinical classification criteria for autoinflammatory periodic fevers. Ann Rheum Dis 2015;74:799-805.

-4 Park H, Bulua Bourla A, Kastner DL, Colbert RA, Siegel RM: Lighting the fires within: the cell biology of autoinflammatory diseases. Nat Rev Immunol 2012;12:570-580.

5 Vogelgesang S, Pottathil V, Robinson JA, Vogelgesang S, Pottathil V, Robinson JA, Rheumatology in the ICU; in Hall JB, Schmidt GA, Kress JP, Hall JB, Schmidt GA, Kress JP (eds): Principles of Critical Care, 4 ed. New York, McGraw-Hill, 2015, chapt 126.

6 Borazan NH, Furst DE, Nabeel H: Nonsteroidal anti-inflammatory drugs, disease-modifying antirheumatic drugs, nonopioid analgesics, and drugs used in gout; in: Katzung BG, Trevor AJ (eds): Basic and Clinical Pharmacology, 13 ed. New York, McGraw-Hill, 2015, chapt 36. 


\section{Case Reports in Neurology}

\begin{tabular}{l|l}
\hline Case Rep Neurol 2017;9:91-97 \\
\hline DOI: 10.1159/000472147 & $\begin{array}{l}\text { (c) 2017 The Author(s). Published by S. Karger AG, Basel } \\
\text { www.karger.com/crn }\end{array}$ \\
\hline
\end{tabular}

Novroski and Baldwin: Chronic Autoimmune Meningoencephalitis and Periodic Fever Syndrome Treated with Anakinra
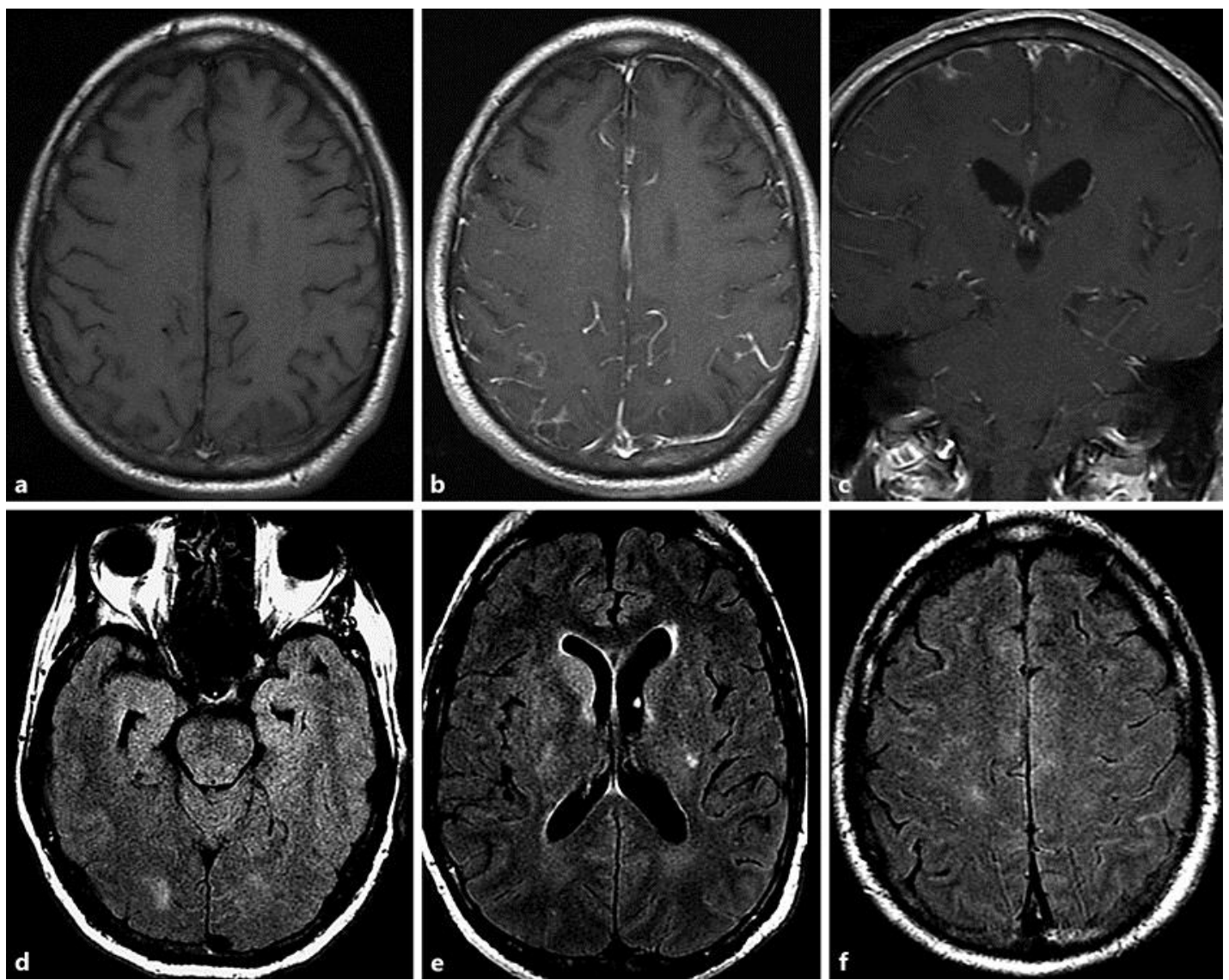

Fig. 1. MRI of the brain with and without contrast. a T1 precontrast axial image without obvious abnormalities. T1 postcontrast axial (b) and coronal (c) images with leptomeningeal enhancement. d-f T2 FLAIR sequence axial cuts with diffuse hyperintensities in the white matter and basal ganglia. 


\section{Case Reports in Neurology}

Case Rep Neurol 2017;9:91-97
\begin{tabular}{l|l}
\hline DOI: $10.1159 / 000472147$ & $\begin{array}{l}\text { (C) 2017 The Author(s). Published by S. Karger AG, Basel } \\
\text { www.karger.com/crn }\end{array}$ \\
\hline
\end{tabular}

Novroski and Baldwin: Chronic Autoimmune Meningoencephalitis and Periodic Fever Syndrome Treated with Anakinra

Table 1. Trend of basic CSF studies over patient hospitalizations

CSF - Basic Studies

\begin{tabular}{|c|c|c|c|c|c|c|c|c|c|}
\hline Collection date (2014) & $1 / 28$ & $2 / 14$ & $4 / 17$ & $5 / 15$ & $5 / 27$ & $6 / 5$ & $6 / 23$ & $7 / 3$ & $12 / 12$ \\
\hline $\mathrm{WBC}$, cells $/ \mathrm{mm}^{3}$ & 380 & 411 & 980 & 27 & 9 & 21 & 11 & 7 & 130 \\
\hline Neutrophils, \% & 46 & 75 & 69 & 22 & 0 cells $/ \mu \mathrm{L}$ & 4 cells $/ \mu \mathrm{L}$ & 3 cells $/ \mu \mathrm{L}$ & 0 cells $/ \mu \mathrm{L}$ & 52 \\
\hline Lymphocytes, \% & 7 & 7 & 4 & 47 & 8 cells $/ \mu \mathrm{L}$ & 7 cells $/ \mu \mathrm{L}$ & 3 cells $/ \mu \mathrm{L}$ & 2 cells $/ \mu \mathrm{L}$ & 32 \\
\hline Monocytes, \% & 47 & 18 & 27 & 31 & 1 cells $/ \mu \mathrm{L}$ & 10 cells $/ \mu \mathrm{L}$ & 5 cells $/ \mu \mathrm{L}$ & 5 cells $/ \mu \mathrm{L}$ & 16 \\
\hline $\mathrm{RBC}$, cells $/ \mathrm{mm}^{3}$ & 8 & 633 & 5 & 0 & 0 & 0 & 292 & 8 & 4 \\
\hline Protein, mg/dL & 126 & 152 & 168 & 108 & 70 & 80 & 61 & 177 & 269 \\
\hline Glucose, mg/dL & 57 & 51 & 50 & 48 & 59 & 56 & 53 & 35 & 51 \\
\hline Xanthochromia & - & - & & & & - & & & - \\
\hline Lactic acid, mmol/L & 3.4 & & 4.2 & & & 2.2 & & & \\
\hline
\end{tabular}

The first date reflects the initial LP. WBC, white blood cells; RBC, red blood cells. 